Female Pelvic Med Reconstr Surg. 2016 ; 22(4): 236-242. doi:10.1097/SPV.0000000000000264.

\title{
Factors Influencing Selection of Vaginal, Open Abdominal, or Robotic Surgery to Treat Apical Vaginal Vault Prolapse
}

\author{
Dr. Mallika Anand, MD, Ms. Amy L. Weaver, MS, Ms. Kristin M. Fruth, BS, and Dr. John B. \\ Gebhart, MD, MS \\ Division of Gynecologic Surgery (Drs Anand and Gebhart) and Division of Biomedical Statistics \\ and Informatics (Mss Weaver and Fruth), Mayo Clinic, Rochester, Minnesota
}

\begin{abstract}
Objectives-To determine factors influencing selection of Mayo-McCall culdoplasty (MMC), open abdominal sacrocolpopexy (ASC), or robotic sacrocolpopexy (RSC) for posthysterectomy vaginal vault prolapse.
\end{abstract}

Methods-We retrospectively searched for the records of patients undergoing posthysterectomy apical vaginal prolapse surgery between January 1, 2000, and June 30, 2012, at our institution. Baseline characteristics and explicit selection factors were abstracted from the electronic medical records. Factors were compared between groups using $\chi^{2}$ tests for categorical variables, ANOVA for continuous variables, and Kruskal-Wallis tests for ordinal variables.

Results-Among the 512 patients identified who met inclusion criteria, the MMC group $(\mathrm{n}=174)$ had more patients who were older, American Society of Anesthesiologists class 3+ or greater, had anterior vaginal prolapse grade $3+$, desired to avoid abdominal surgery, and did not desire a functional vagina. Patients in the ASC $(n=237)$ and RSC $(n=101)$ groups had more failed prolapse surgeries, suspected abdominopelvic pathologic processes, and chronic pain. Advanced prolapse was more frequently cited as an explicit selection factor for ASC than for either MMC or RSC.

Conclusions-The most common factors that influenced the type of apical vaginal vault prolapse surgery overlapped with characteristics that differed at baseline. In general, MMC was chosen for advanced anterior vaginal prolapse and baseline characteristics that increased surgical risks, ASC for advanced apical prolapse, and ASC or RSC for recurrent prolapse, suspected abdominal pathology, and patients with chronic pain or lifestyles including heavy lifting. Thus, efforts should be made to attempt to control for selection bias when comparing these procedures.

\section{Keywords}

apical prolapse; Mayo-McCall culdoplasty; posthysterectomy vaginal prolapse; sacrocolpopexy

Reprints: John B. Gebhart, MD, MS, Division of Gynecologic Surgery, Mayo Clinic, 200 First St SW, Rochester, MN 55905, (gebhart.john@mayo.edu, Phone: [507] 266-7711, Fax: [507] 266-9300).

Presented at the Society of Gynecologic Surgeons 41st Annual Scientific Meeting, Orlando, Florida, March 22-25, 2015.

Conflict of Interest: Dr Gebhart serves as a Consultant for AMS and has received royalties from UpToDate and Elsevier. 


\section{Introduction}

Apical vaginal vault prolapse affects $1.8 \%$ to $11.6 \%$ of women who have undergone hysterectomy (1). Symptomatic vaginal vault prolapse can cause bothersome symptoms associated with the vaginal bulge and the adjacent bladder and bowel, which decrease overall quality of life (2). Treatment options for vaginal vault prolapse include observation, pessary, or surgery (3). Surgery can be approached via the transvaginal or transabdominal routes and can use various techniques with each route. Transvaginal approaches include procedures that preserve the vagina, as well as obliterative procedures. Transvaginal approaches include mesh-augmented repairs and native tissue repairs comprising variations of uterosacral ligament suspension and sacrospinous ligament fixation. Obliterative transvaginal approaches consist of colpocleisis and colpectomy. The most common transabdominal procedure is abdominal sacrocolpopexy. Minimally invasive transabdominal procedures that have been more recently developed include laparoscopic sacrocolpopexy and robotic sacrocolpopexy $\left(3^{-} 5\right)$.

Determining which approach to use for a given patient can be challenging, and there is no clear consensus to guide this decision. According to the classic, evidence-based textbook, Urogynecology and Reconstructive Pelvic Surgery (3), if the vaginal apex descends less than half of the total vaginal length, vaginal approaches can generally alleviate vaginal dysfunction and symptoms; however, there is little evidence to guide the route of surgery in cases of advanced vaginal vault prolapse. In addition to the degree of apical prolapse, factors influencing selection of surgical approach can include the surgeon's training, patient preference, comorbid conditions, coexisting pathologic processes, and additionally planned prolapse procedures $(3,5,6)$.

Three surgical procedures for vaginal vault prolapse are performed at our institution: vaginal Mayo-McCall culdoplasty (MMC), open abdominal sacrocolpopexy (ASC), and minimally invasive transabdominal robotic sacrocolpopexy (RSC). The objective of the current study was to determine factors influencing selection of surgical procedure for posthysterectomy vaginal vault prolapse at our institution. We hypothesized that patient baseline characteristics would differ among the 3 surgical types and that explicitly stated factors in the preoperative consultation documents would also influence selection of surgical route.

\section{Methods}

This was a single-institution, retrospective cohort study, and the Mayo Clinic Institutional Review Board approved the protocol. Patients were identified using the Surgical Operative Note Explorer and were included if they underwent posthysterectomy vaginal vault prolapse repair via MMC, ASC, or RSC at Mayo Clinic, Rochester, Minnesota, between January 1, 2000, and June 30, 2012. The Division of Gynecologic Surgery began to perform RSC in 2007. Patients who did not allow access to their medical records for research purposes were excluded. Patients were also excluded if they had prior or concomitant vaginal mesh excision, upper vaginectomy, nonhealing vaginal wound, radical hysterectomy, oncologic surgery, systemic chemotherapy, pelvic radiation therapy, current malignancy, connective tissue disease, nonpermanent mesh, concomitant trachelectomy or hysterectomy, 
concomitant major nongynecologic surgery (rectopexy, colectomy), suspension of neovagina, fistula repair, pouch of Douglas hernia, or no apical prolapse, or if their procedure was performed by a nongynecologic surgeon or otherwise misclassified.

All data were abstracted from the electronic health record. Study data were collected and managed using REDCap electronic data capture tools hosted at Mayo Clinic (7). Patient baseline characteristics abstracted included age, ethnicity, body mass index, smoking status, vaginal parity, comorbid conditions, American Society of Anesthesiologists (ASA) physical classification status, number and type of prior pelvic organ prolapse surgeries, number and type of prior urinary incontinence surgeries, pelvic floor symptoms (eg, bladder dysfunction, bowel dysfunction, and dyspareunia), chronic pain of any kind, and preoperative BadenWalker prolapse grade of each vaginal compartment (8). A Charlson comorbidity index score for longitudinal morbidity was assigned on the basis of comorbid conditions (9). To abstract all explicitly stated factors for selection of route of surgery, the "Impression, Report, and Plan" section of the preoperative consultation notes were reviewed. Intraoperative variables consisting of surgeon and primary and concomitant surgeries were confirmed. All surgeons were fellowship trained in urogynecology (4 surgeons, $90.8 \%$ of the surgeries) or gynecologic oncology (7 surgeons, $9.2 \%$ of the surgeries). Procedures performed concomitantly with apical prolapse surgery were also abstracted.

Statistical analyses were performed using SAS version 9.2 software (SAS Institute, Inc). Patients were grouped according to type of surgery: MMC, ASC, or RSC. Baseline characteristics were compared among these 3 procedure groups using the $\chi^{2}$ test for categorical (nonordered) variables, the F-test from a 1-way analysis of variance model for age and body mass index, and the Kruskal-Wallis test for all other ordinal variables. Explicitly stated factors influencing selection contained in the consultation notes were summarized. Factors that were stated differently but that shared an underlying rationale were combined (eg, "multiply operated vagina" and "short vaginal length"). The explicitly stated factors, and each of the concomitant procedures, were compared between the 3 procedures using the $\chi^{2}$ test or Fisher exact test, as appropriate. All calculated $P$ values were 2-sided, and $P<.05$ was considered statistically significant. If a $P$ value was $<.05$ for an overall test for differences between any of the 3 procedures, pairwise comparisons between the 3 procedures were further evaluated without adjustment for multiple comparisons.

\section{Results}

A total of 711 charts were identified and assessed for eligibility (252 MMC, 349 ASC, and 110 RSC). Thirty patients did not provide research authorization. An additional $65 \mathrm{MMC}$, 100 ASC, and 4 RSC patients met exclusion criteria. The resulting cohort comprised 512 patients in the 3 groups: $174 \mathrm{MMC}, 237 \mathrm{ASC}$, and $101 \mathrm{RSC}$. The relative proportions of the 3 procedures varied by year (Figure). In 2007, 62.9\% of the procedures were ASC and $11.4 \%$ were RSC. By 2011 (the last full calendar year), both ASC and RSC were $36.1 \%$. As the proportion of robotic procedures increased, the proportion of abdominal procedures decreased, whereas the proportion of vaginal procedures overall (MMC) remained stable. For the 2 surgeons who performed most of the RSCs (as an option in addition to ASC), the 
relative proportion of RSC to ASC generally increased between 2007 and 2011; the proportion of MMC was stable during the same 5-year period for these 2 surgeons.

Mean age of the entire cohort at the time of surgery was 63.2 \pm 10.9 years (range, 34-94 years). Patients in the MMC group were significantly older (mean, 68.9 years) than patients in the ASC and RSC groups (60.4 and 59.9 years) (both $P<.001)$ and were more likely than the other 2 groups to have a classification of ASA 3+ (MMC, $25.2 \%$ vs ASC, $11.8 \%$, and vs RSC, 8.9\%; both $P<.001$ ) (Table 1). More patients undergoing sacrocolpopexy had prior prolapse surgeries, and prior vaginal prolapse surgeries in particular (ASC, 62.9\%; RSC, $61.4 \%$ ), compared with the MMC group (35.6\%; both $P<.001)$. A greater proportion of patients in the RSC group had prior sling placement for stress urinary incontinence (24.8\%) than did patients in the MMC (8.6\%) or ASC (8.4\%) groups (both $P<.001)$. The RSC group had less need to splint to defecate or a sensation of incomplete bowel evacuation (6.9\%) compared with the MMC (23.0\%; $P<.001)$ or ASC (19\%; $P=.005)$ group. Patients in the RSC group had less anal incontinence at baseline (5.9\%) compared with the MMC group $(14.9 \% ; P=.03)$. Patients undergoing either type of sacrocolpopexy were more likely to have underlying chronic pain of any kind than were patients undergoing MMC (ASC, 15.2\% vs MMC, 3.4\%, $P<.001$; RSC, $11.9 \%$ vs MMC, 3.4\%, $P=.006)$.

With regard to prolapse grade, patients in the ASC group were significantly more likely to have advanced (grade 3 or 4) apical prolapse at baseline compared with either MMC or RSC (both $P<.001$ ) (Table 1). Patients in the MMC group had a greater proportion of advanced (grade 3 or 4$)$ anterior vaginal compartment prolapse than either ASC $(P<.001)$ or RSC $(P=$ 004). Roughly one-third of patients in both the MMC and ASC groups had grade 3 or 4 rectocele at baseline, about twice as many as among the RSC group (MMC vs RSC, $P=.004$; ASC vs RSC, $P=.002$ ).

In terms of explicitly stated selection factors, a large proportion of patients did not have any explicitly stated reasons in their health record for selection of route of surgery (Table 2). Several factors were only rarely cited, and in some cases, more than 1 factor was stated for a given patient. "Patient preference" was cited as a reason for selection in slightly more than half of the RSC group. Young age was a factor cited among the ASC group (11.0\%). Factors significantly associated with selection of abdominal or robotic sacrocolpopexy included multiply operated vagina, shortened vaginal length, prior failed prolapse surgeries, increased existing or anticipated Valsalva pressure, and desire to avoid open abdominal surgery (all $P<.001)$. Increased degree of prolapse was explicitly stated with greater frequency among the ASC than the MMC group ( $P=.049)$, as was need for abdominal exploration or presence of abdominal hernia $(P=.02)$. Compared with MMC and ASC, patients undergoing RSC had a more frequent explicitly stated selection factor of pelvic pain (RSC, 5.0\% vs MMC, $0 \%$ $[P=.006]$ and ASC, $1.3 \%[P=.05])$. Sexual inactivity or lack of desire for a functional vagina was frequently cited as a selection factor for MMC (12.6\%).

Several infrequent, although interesting, reasons cited for selection of a particular route in general or over another included desire to avoid mesh (3 MMC patients), isolated apical prolapse (3 sacrocolpopexy patients), and desire to avoid interfering with previously placed vaginal mesh (1 ASC patient). For 1 patient, an enterocele could not be reduced vaginally, so 
the decision was made to proceed with an abdominal approach. For 2 patients with voiding dysfunction, ASC was chosen with the aim to augment bladder emptying. Three patients underwent ASC as opposed to RSC with the specific desire to avoid RSC because it was a relatively new approach or because of concern for possible preexisting adhesions after multiple prior abdominal surgeries. Concomitant procedures differed significantly among the 3 groups (Table 3 ). The MMC group had the greatest proportion with either concomitant anterior $(82.8 \%)$ or posterior $(91.4 \%)$ repair.

\section{Discussion}

In this descriptive study, we found that, compared with patients undergoing ASC or RSC, patients undergoing MMC were more likely to be older, have a higher ASA score, and have a greater degree of associated anterior vaginal compartment prolapse and were less likely to desire vaginal function at baseline. Factors associated with selection of sacrocolpopexy were more prior prolapse surgeries, multiply operated vagina, chronic pain, and concern for chronic intra-abdominal pressure. Advanced apical prolapse constituted a selection factor for ASC only. Although patients in the MMC group underwent posterior repair with the greatest frequency, repair of a rectocele, if one persisted after correction of the vaginal apex intraoperatively, would have been possible in all groups, including ASC and RSC. Thus, the reason for more frequent rectocele repair in the MMC group, followed by the ASC group, is unclear. It is possible that 1) intraoperative examination altered the degree of prolapse in a given compartment, 2) a primary vaginal approach (MMC) may have afforded more ready access to correct smaller rectoceles, 3) ASC or RSC reduced the posterior compartment to a greater degree, or 4) rectocele repair was avoided in patients with pain, a characteristic more prevalent among ASC and RSC patients.

Among sacrocolpopexy procedures, we found that the relative proportion of ASC decreased with increased adoption of the RSC technique. This suggests that, rather than supplanting the primary vaginal approach, RSC may have been supplanting ASC. As minimally invasive approaches continue to develop, it will be essential to continue to follow these trends over time.

Relating patient characteristics to the stated factors, nearly all of the statistically significant differences in baseline characteristics among the treatment groups were reflected in the most commonly cited explicit selection factors. The main exception to this was that the greater proportion of advanced (grade 3 or 4 ) anterior vaginal compartment prolapse in the MMC group at baseline was not reflected in explicitly stated selection factors. Conversely, some explicitly stated selection factors, such as "patient desires to avoid abdominal surgery" were not captured among baseline characteristics.

There are few formal reports on preference for different routes of apical vaginal prolapse repair $(6,10,11)$. In a 2007 survey-based study of surgeons in the United Kingdom and Australia, Vanspauwen et al (10) found that older patient age appeared to increase preference for transvaginal repair of posthysterectomy vaginal vault prolapse. Jha and Moran (11) found that among physician survey respondents in the United Kingdom, 11\% would change their procedure of choice for posthysterectomy vaginal vault prolapse if the patient

Female Pelvic Med Reconstr Surg. Author manuscript; available in PMC 2016 July 01. 
were not sexually active. In a survey of gynecologic surgeons regarding factors influencing selection of repair type for primary and recurrent apical vaginal prolapse, Montoya et al (6) found that the transvaginal native tissue approach was more common for primary apical repair, whereas minimally invasive transabdominal approaches were used for recurrent prolapse. In that study, the most frequently cited factors that surgeons considered for primary or recurrent apical vaginal prolapse were desire to maintain vaginal function, age, comorbid conditions, and apical prolapse stage, without quantification of directional effects. Surgeons preferring a laparoscopic route of repair (laparoscopic sacrocolpopexy, robotic sacrocolpopexy, or laparoscopic uterosacral ligament suspension) cited prolapse stage and surgeon experience as important considerations (6). Our findings were similar in that prolapse stage affected selection, in addition to patient age and health status (in our case correlating with ASA score, not Charlson comorbidity score). In our study, advanced anterior vaginal prolapse, found to differ at baseline, may have influenced selection of the vaginal approach.

Strengths of our study include a large cohort of patients undergoing surgery for a primary indication of posthysterectomy vaginal vault prolapse. Internal validity was further strengthened by the limited types of prolapse surgeries offered and by all surgeons being fellowship trained in gynecologic surgery at the same institution. Additionally, rather than surveying surgeons themselves regarding preference, we sought to determine possible selection factors on the basis of patient characteristics and documented rationale in the medical records.

Limitations of our study are that it is a retrospective study from a single institution. Although we detected several statistically significant factors possibly influencing selection, the study was not powered, a priori, to detect differences for all potential selection factors. Finally, given that consultation notes were open ended, the factor(s) influencing selection might not always have been explicitly stated.

Ultimately, although there was some overlap, we saw some notable differences in the selection of surgical procedure for vaginal vault prolapse. In general, surgeons chose MMC for patients with more advanced anterior vaginal prolapse and baseline characteristics that increased the risks of surgery. Surgeons chose ASC for more advanced apical prolapse and chose ASC or RSC for recurrent prolapse, for suspected concurrent abdominal pathology, and for patients with chronic pain or lifestyles that included heavy lifting. Furthermore, between ASC and RSC procedures, the proportion of RSC changed over time as this procedure became increasingly adopted. It is thus imperative that comparative studies of vaginal vault prolapse, especially nonrandomized cohort studies, attempt to control for selection bias when drawing conclusions.

\section{Acknowledgments}

Source of Funding: This research was supported by Grant Number UL1 TR000135 from the National Center for Advancing Translational Sciences (NCATS) and internal funding from the Division of Gynecologic Surgery, Department of Obstetrics and Gynecology, Mayo Clinic, Rochester, MN. The data analysis was supported by funds from the Mayo Foundation. 


\section{Abbreviations}
ASA American Society of Anesthesiologists
ASC open abdominal sacrocolpopexy
MMC Mayo-McCall culdoplasty
RSC transabdominal robotic sacrocolpopexy

\section{References}

1. Marchionni M, Bracco GL, Checcucci V, Carabaneanu A, Coccia EM, Mecacci F, et al. True incidence of vaginal vault prolapse: thirteen years of experience. J Reprod Med. 1999 Aug; 44(8): 679-84. [PubMed: 10483537]

2. Rortveit G, Brown JS, Thom DH, Van Den Eeden SK, Creasman JM, Subak LL. Symptomatic pelvic organ prolapse: prevalence and risk factors in a population-based, racially diverse cohort. Obstet Gynecol. 2007 Jun; 109(6):1396-403. [PubMed: 17540813]

3. Walters, MD.; Karram, MM., editors. Urogynecology and Reconstructive Pelvic Surgery. 3. Philadelphia (PA): Mosby Elsevier; c2007. Surgical treatment of vaginal vault prolapse and enterocele; p. 262-87.

4. Diwadkar GB, Barber MD, Feiner B, Maher C, Jelovsek JE. Complication and reoperation rates after apical vaginal prolapse surgical repair: a systematic review. Obstet Gynecol. 2009 Feb; $113(2$ Pt 1):367-73. Erratum in: Obstet Gynecol. 2009 Jun;113(6):1377. [PubMed: 19155908]

5. Maher C, Feiner B, Baessler K, Schmid C. Surgical management of pelvic organ prolapse in women. Cochrane Database Syst Rev. 2013 Apr.30:4, CD004014.

6. Montoya TI, Grande KB, Rahn DD. Apical vaginal prolapse surgery: practice patterns and factors guiding route of repair. Female Pelvic Med Reconstr Surg. 2012 Nov-Dec;18(6):315-20. [PubMed: 23143421]

7. Harris PA, Taylor R, Thielke R, Payne J, Gonzalez N, Conde JG. Research electronic data capture (REDCap): a metadata-driven methodology and workflow process for providing translational research informatics support. J Biomed Inform. 2009 Apr; 42(2):377-81. Epub 2008 Sep 30. [PubMed: 18929686]

8. Baden WF, Walker TA. Genesis of the vaginal profile: a correlated classification of vaginal relaxation. Clin Obstet Gynecol. 1972 Dec; 15(4):1048-54. [PubMed: 4649139]

9. Charlson ME, Pompei P, Ales KL, MacKenzie CR. A new method of classifying prognostic comorbidity in longitudinal studies: development and validation. J Chronic Dis. 1987; 40(5):37383. [PubMed: 3558716]

10. Vanspauwen R, Seman E, Dwyer P. Survey of current management of prolapse in Australia and New Zealand. Aust N Z J Obstet Gynaecol. 2010 Jun; 50(3):262-7. [PubMed: 20618245]

11. Jha S, Moran P. The UK national prolapse survey: 5 years on. Int Urogynecol J. 2011 May; 22(5): 517-28. Epub 2011 Feb 22. [PubMed: 21340642] 


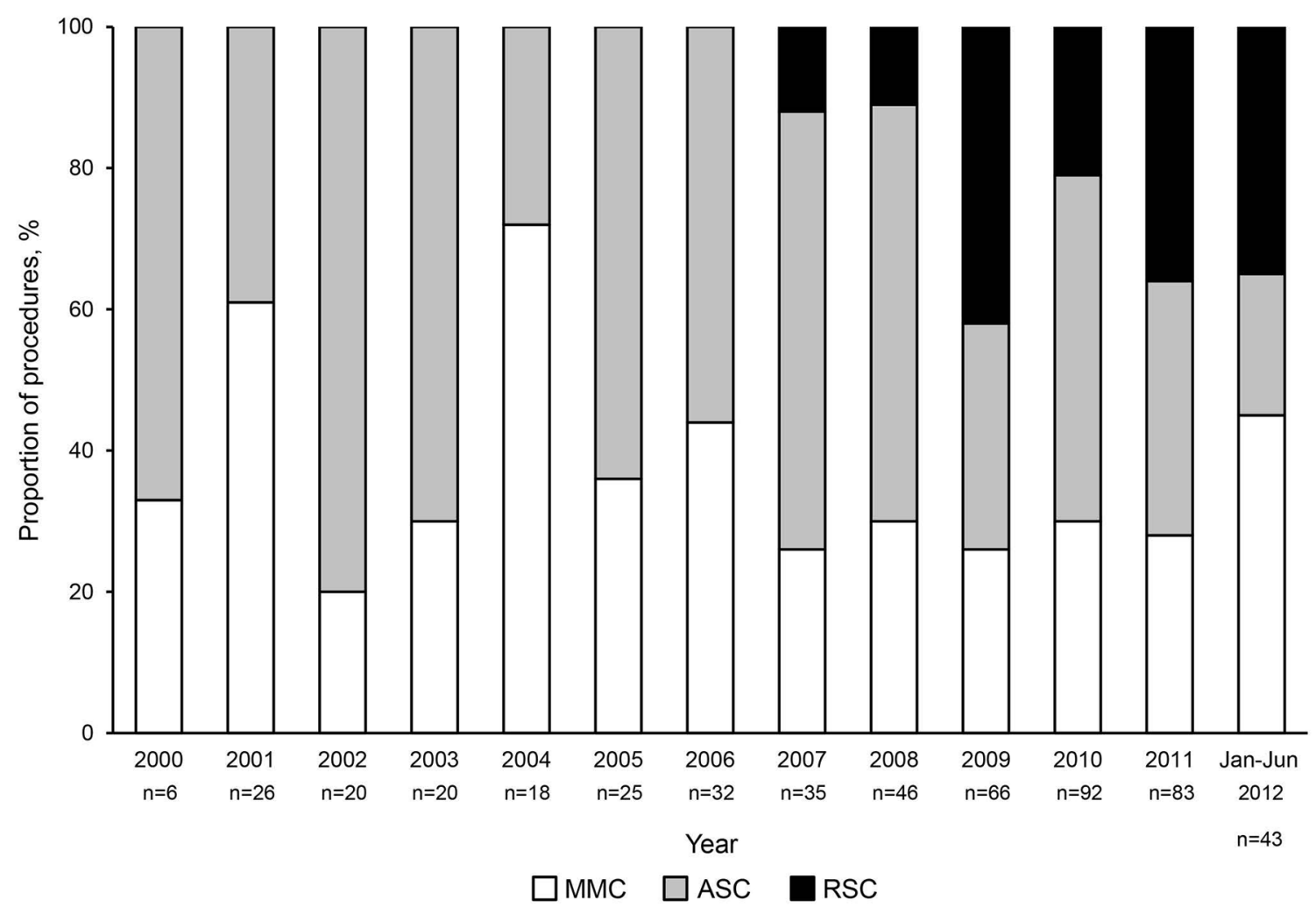

Figure.

Proportion of Procedures by Year. Proportion of procedures performed with open abdominal sacrocolpopexy (ASC), Mayo-McCall culdoplasty (MMC), and transabdominal robotic sacrocolpopexy (RSC) from 2000 to 2012. Use of RSC began in our department in 2007. 


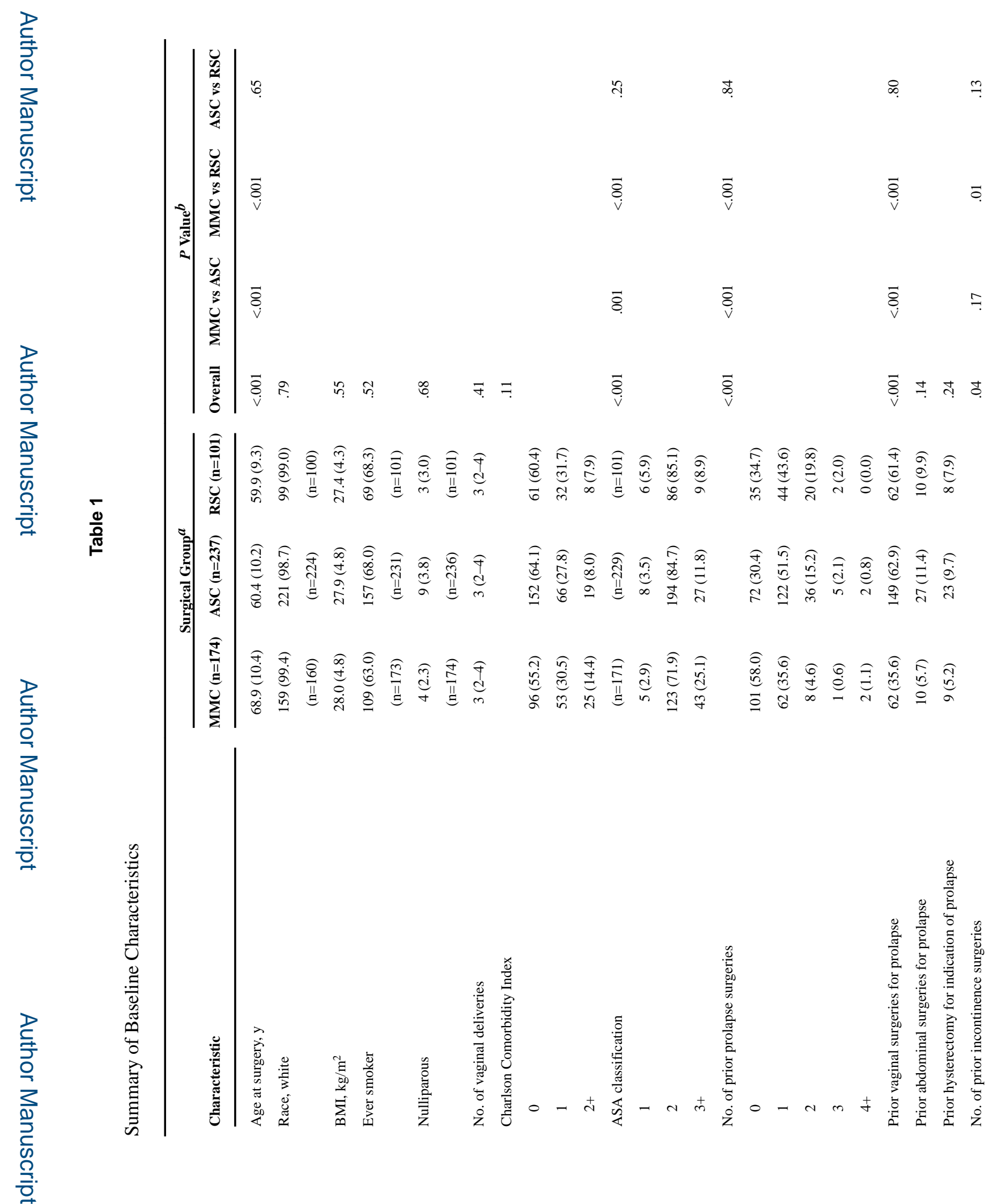

Female Pelvic Med Reconstr Surg. Author manuscript; available in PMC 2016 July 01. 
Anand et al.

Page 10

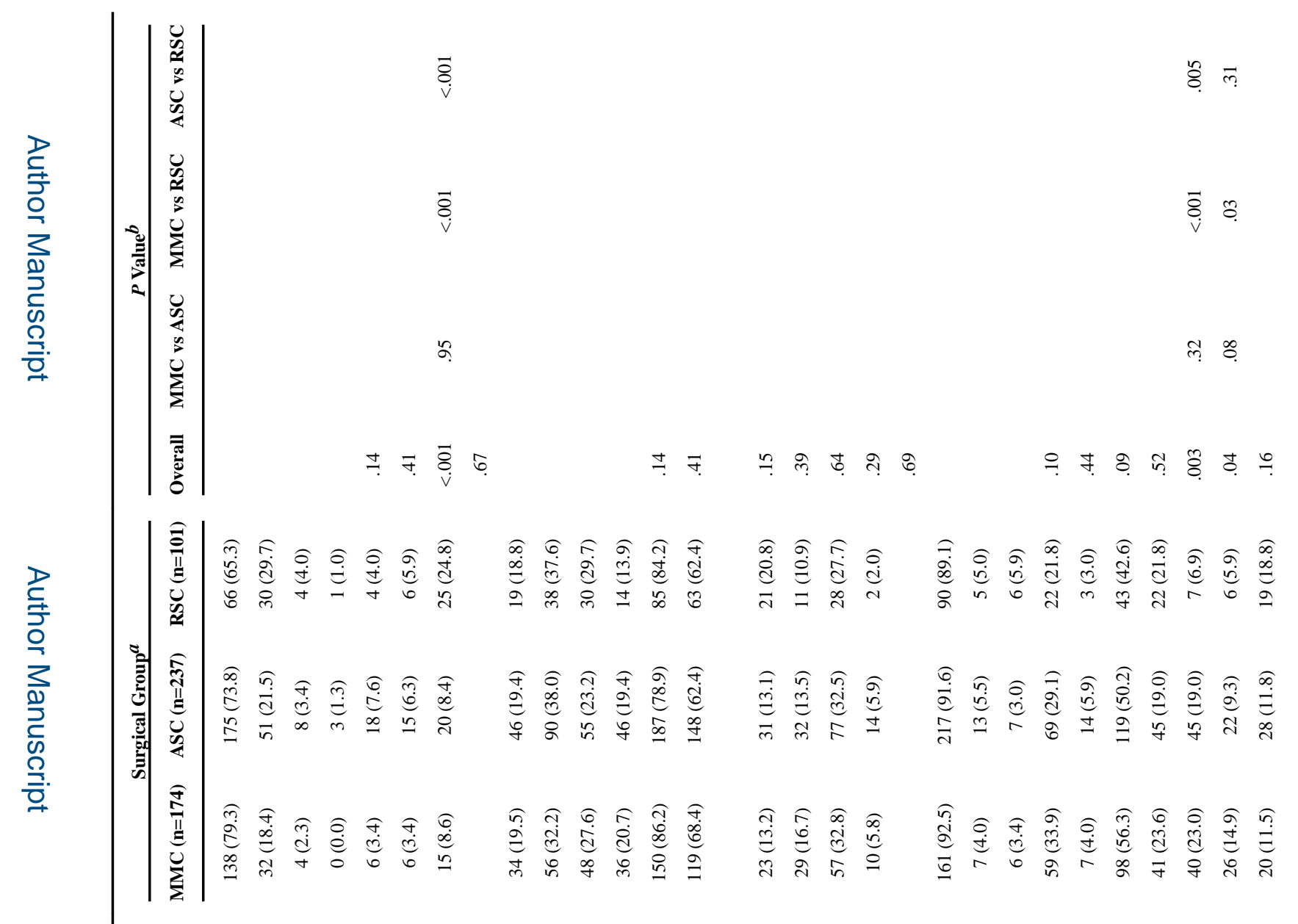

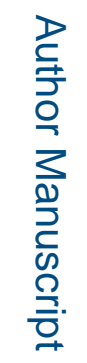

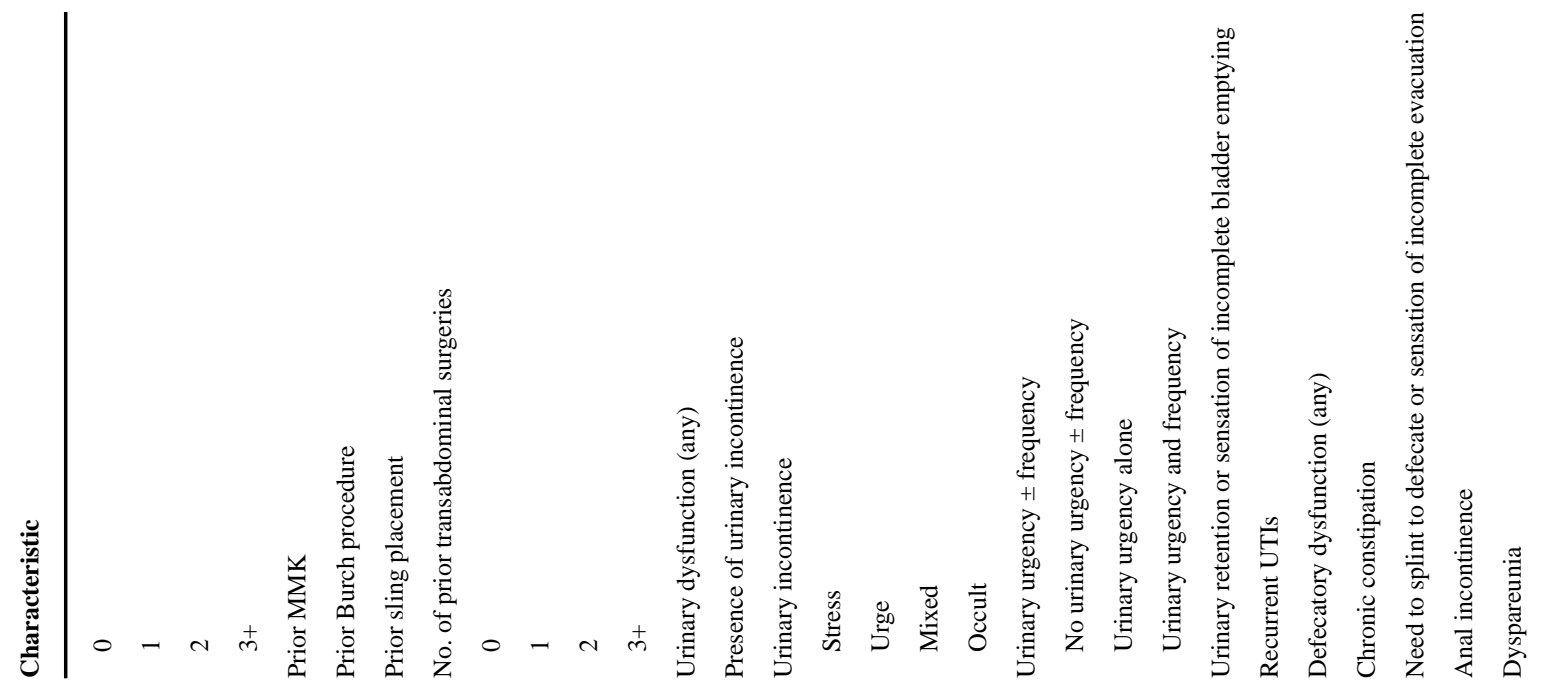

Female Pelvic Med Reconstr Surg. Author manuscript; available in PMC 2016 July 01. 


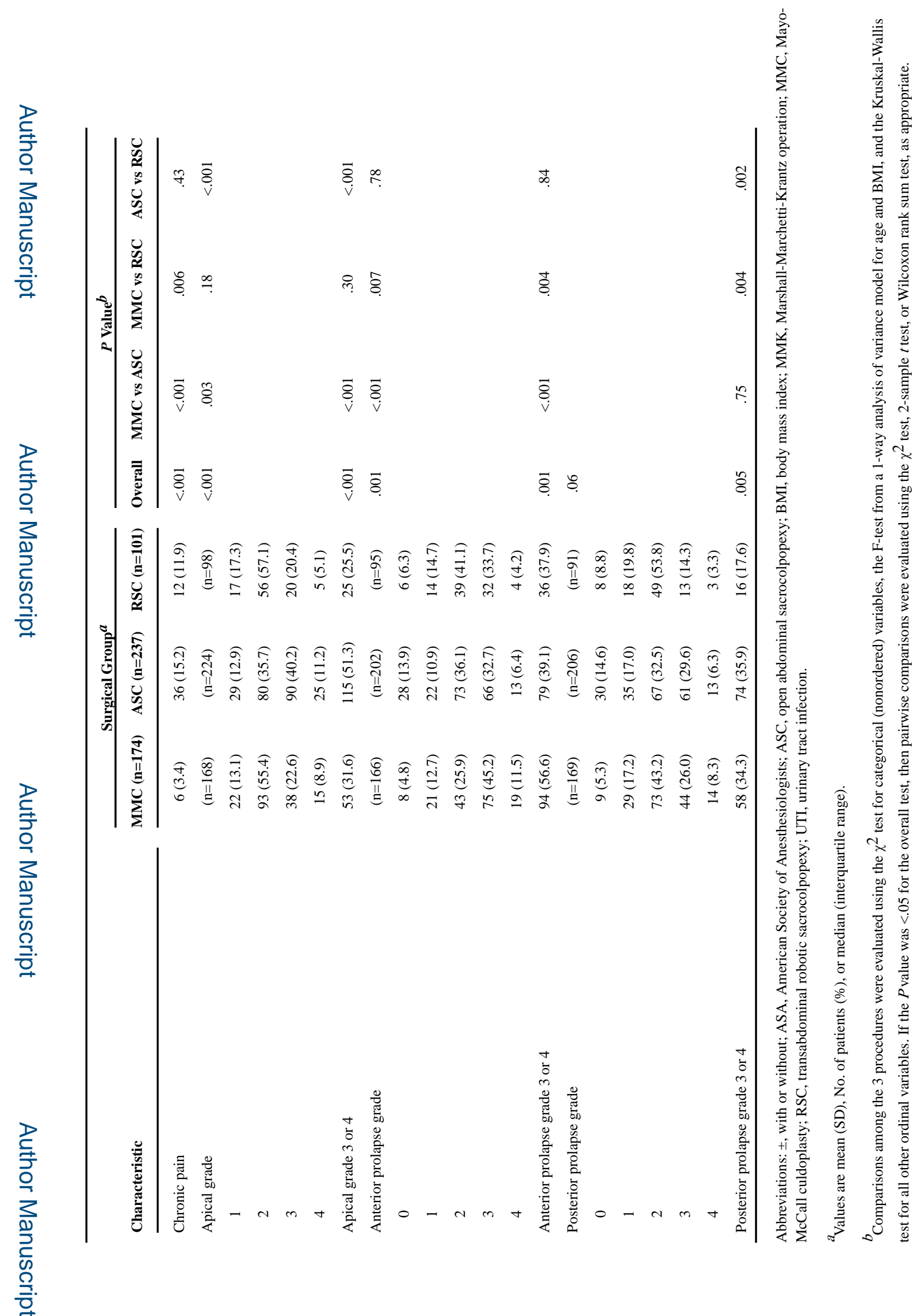

Female Pelvic Med Reconstr Surg. Author manuscript; available in PMC 2016 July 01. 


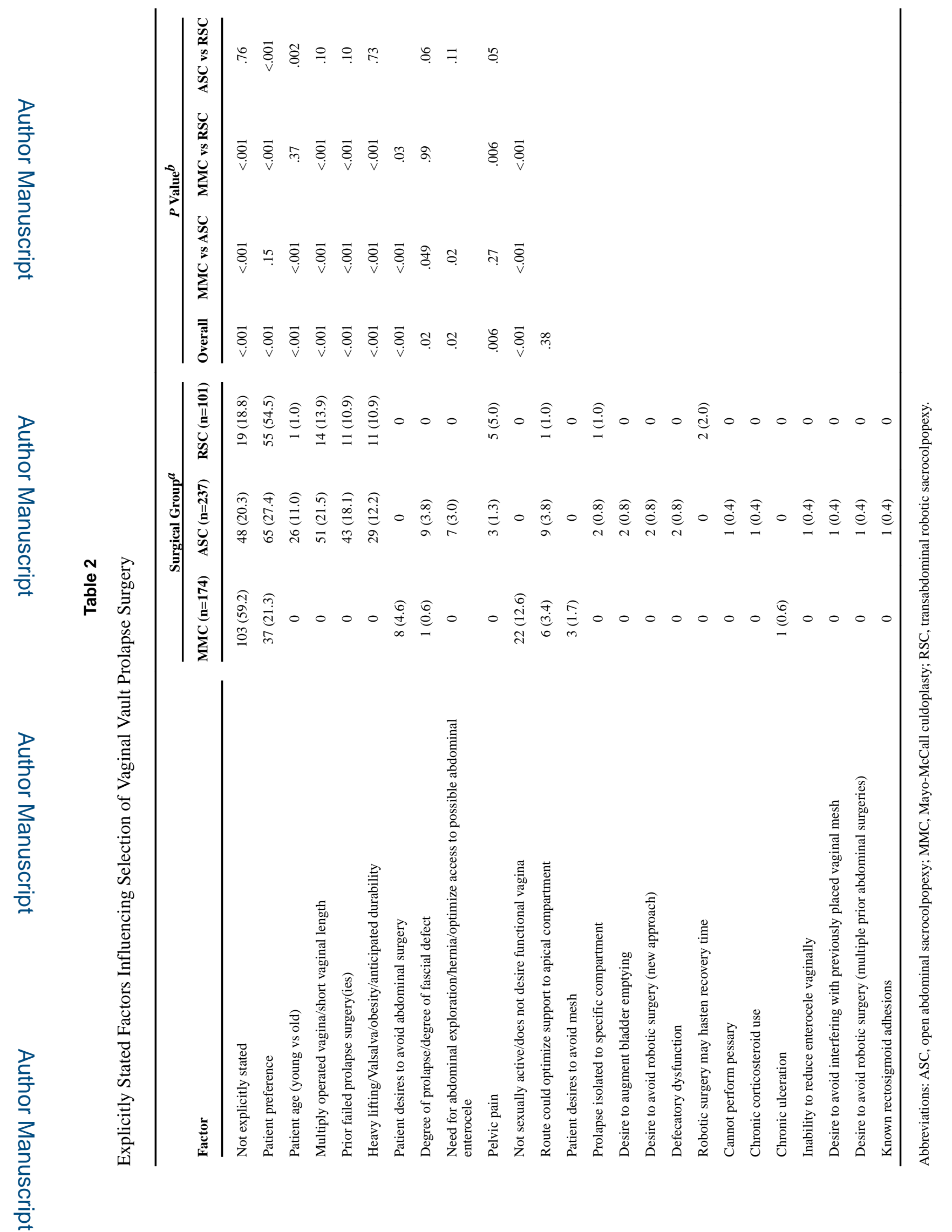

Female Pelvic Med Reconstr Surg. Author manuscript; available in PMC 2016 July 01. 


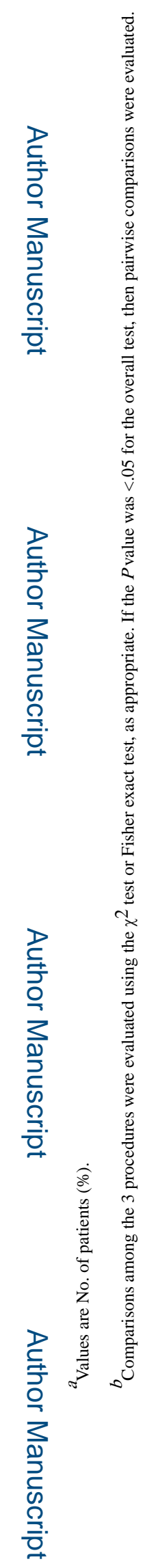




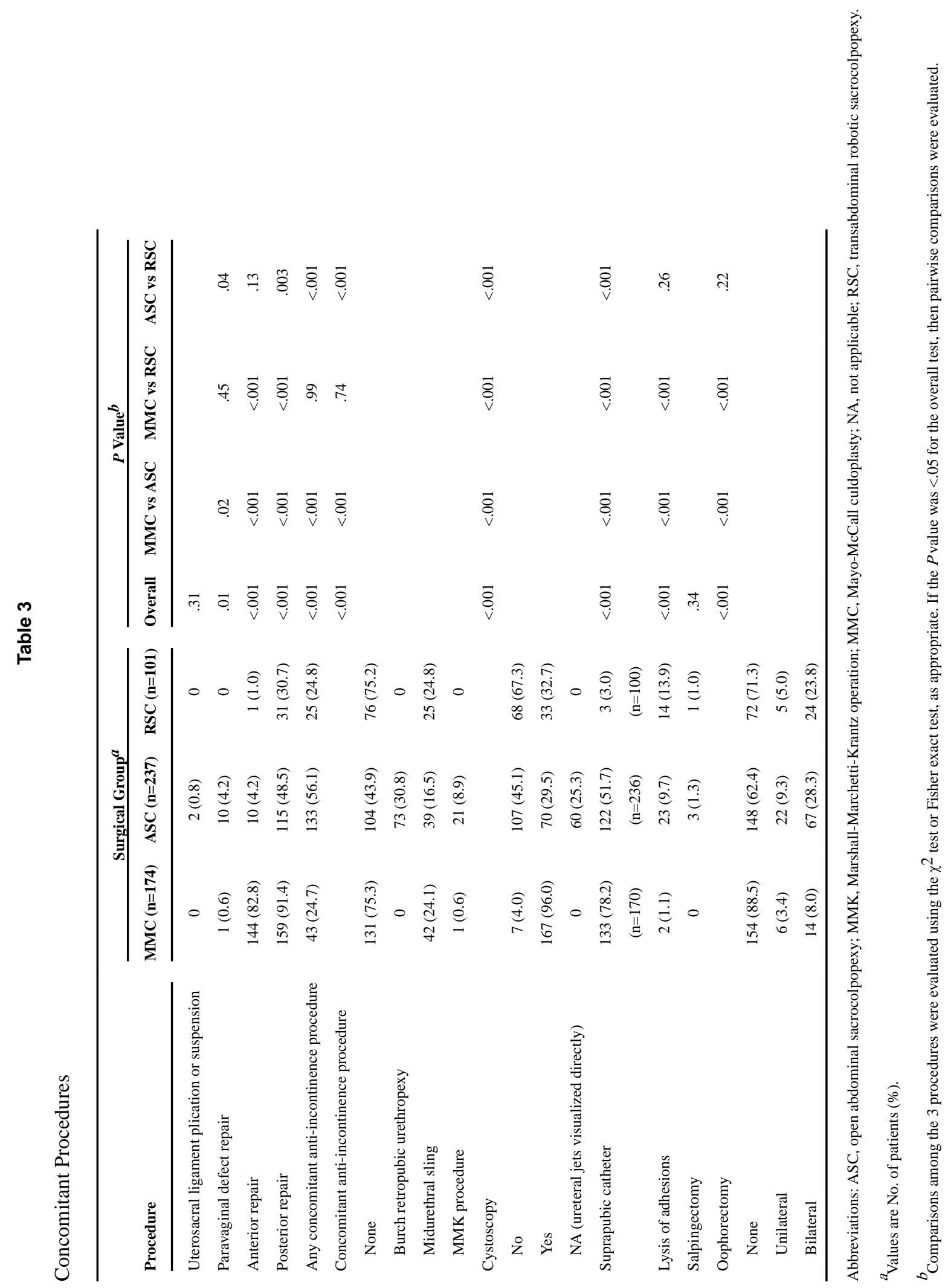

Female Pelvic Med Reconstr Surg. Author manuscript; available in PMC 2016 July 01. 